Research Article

\title{
High-Score US-Suspicious Subcentimeter Thyroid Nodules: What Factors Affect Adequate Sampling of US-Guided Fine-Needle Aspiration Biopsy?
}

\author{
Yang Li $\mathbb{D}^{1},{ }^{1}$ Jin Hong Yu $\mathbb{C}^{2},{ }^{2}$ Ping Jie Du ${ }^{\mathbb{D}},{ }^{1}$ Yu Xie $\mathbb{C},{ }^{2}$ Sushant Kumar Das $\mathbb{D}^{1},{ }^{1}$ Bing Li, ${ }^{1}$ \\ and Chuan Zhang $\mathbb{1}^{1}$ \\ ${ }^{1}$ Department of Radiology, The Affiliated Hospital of North Sichuan Medical College, 63 Wenhua Road, Nanchong 637000, \\ Sichuan, China \\ ${ }^{2}$ Department of Ultrasound, The Affiliated Hospital of North Sichuan Medical College, 63 Wenhua Road, Nanchong 637000, \\ Sichuan, China
}

Correspondence should be addressed to Jin Hong Yu; 525293623@qq.com

Received 29 January 2020; Accepted 20 March 2020; Published 21 April 2020

Academic Editor: Lodovico Rosato

Copyright (C) 2020 Yang Li et al. This is an open access article distributed under the Creative Commons Attribution License, which permits unrestricted use, distribution, and reproduction in any medium, provided the original work is properly cited.

\begin{abstract}
Background. Fine-needle aspiration biopsy (FNAB) is diagnostic standard for thyroid nodules. However, the influence of adequate sample rate of US-guided FNAB for subcentimeter thyroid nodules is not known well. Objectives. To assess the factors affecting adequate sample rate of US-guided FNAB for subcentimeter thyroid nodules. Methods. Three hundred and forty-nine consecutive US-guided FNAB procedures were performed in 344 patients with subcentimeter thyroid nodules. The adequate sample rate was analyzed for all nodules on the basis of nodule-related and technical factors. The factors affecting adequate sample rate of USguided FNAB for subcentimeter thyroid nodules were determined by multivariate logistic regression. Results. The adequate sample rate increased with larger nodules $(72.7 \%$ for $3-6 \mathrm{~mm}$ nodules and $84.9 \%$ for $7-10 \mathrm{~mm}$ nodules $(P=0.007))$. The adequate sample rate was $63.9 \%, 81.3 \%$, and $90.6 \%$ in nodules with macrocalcifcation, microcalcification, and no calcification, respectively $(P<0.001)$. The adequate sample rate was $71.8 \%$ for biopsies performed with a perpendicular needle path and $85.0 \%$ with a parallel needle path $(P=0.004)$. The significant factors affecting adequate sample rate of US-guided FNAB for subcentimeter thyroid nodules were nodule size $(P<0.001$; odds ratio $(\mathrm{OR})$ for $7-10 \mathrm{~mm}$ nodules was approximately 3.0 times higher than that for 3-6 mm nodules), calcification $(P<0.001$; OR for nodules without calcification was approximately 5.3 times higher than that for the nodules with macrocalcification), and needle path $(P=0.044$; OR for the use of the parallel needle path was about 1.8 times higher than that for the perpendicular needle path). Conclusion. Nodule size, calcification, and needle path were the determinants of sample adequacy. The adequate sample rate was higher in larger nodules, in nodules without calcification, and upon using a parallel needle path for biopsy.
\end{abstract}

\section{Introduction}

Thyroid nodules are a common medical problem. The increasing number of subcentimeter thyroid nodules detected by high-resolution ultrasonography (US) has brought a new problem for management of such subcentimeter nodules. Accurate diagnosis is an important step in the management and treatment of these nodules. US-guided FNAB has been a reliable, safe, and cost-effective method for the differential diagnosis of benign and malignant thyroid nodules [1-4]. The primary purpose of FNAB is to promote the disease management process and determine whether surgery is needed or not. The reported diagnostic accuracy of FNAB is well-established and ranges from $70.2 \%$ to $97 \%$ [5-10]. However, an inevitable limitation of US-guided FNAB is a nondiagnostic sample, which range in rate from $4.2-40 \%[8,11-13]$. There are many factors that affect FNAB results, such as nodule size, ultrasound features, sampling technique, needle size, and so on $[8,10,13-15]$. 
The clinical significance of thyroid papillary microcarcinoma remains controversial; therefore, how to deal with subcentimeter thyroid nodules properly has caused widespread debate [16] The guidelines recommend that FNAB should be considered for nodules less than $1 \mathrm{~cm}$ only if these nodules have suspicious ultrasound features, such as hypoechoic nodule, microcalcifications, irregular border, and taller than wide shape $[17,18]$. Several studies have been carried out to examine the benefits of US-guided FNAB of subcentimeter thyroid nodules [8, 10, 13, 16, 19], and some experts believe that the combination of suspicious ultrasound features merits referral for US-guided FNAB $[8,19]$. However, to our knowledge, there has been no report that has evaluated factors affecting the adequate sample rate of US-guided FNAB for subcentimeter thyroid nodules.

The objective of our study was to retrospectively identify the factors, in relation to lesion characteristics and the biopsy technique, influencing the adequate sample rate of USguided FNAB for subcentimeter thyroid nodules.

\section{Materials and Methods}

2.1. Patients. From January 2016 to October 2018, 3720 thyroid nodules underwent US-guided FNABs in our institution. Among them, 349 nodules $(9.4 \%, 349 / 3720)$ in 344 patients ( 81 men, 263 women; range: $17-78$ years, mean age of $45.4 \pm 12.3$ years) were subcentimeter thyroid nodules. Five patients underwent biopsy for two different nodules. The medical records, ultrasound findings, and cytologic results or/and histologic results in all patients were retrospectively reviewed.

In our practice, the reasons for US-guided FNAB of subcentimeter thyroid nodules were multifactorial. The inclusion criteria were as follows: (a) a thyroid nodule larger than $5 \mathrm{~mm}$ with probable malignant assessment on US, (b) a thyroid nodule larger than $5 \mathrm{~mm}$ in the patient with highrisk history, and (c) a thyroid nodule in the case of suspected cervical lymph node metastasis $[11,17,19]$. Thyroid nodules were evaluated by using the Thyroid Imaging Reporting and Data System (TI-RADS). Nodules with TI-RADS category $4 \mathrm{~A}$ and above were considered suspicious lesions. Suspicious malignant US findings included a solid hypoechoic structure, an ill-defined margin, microcalcifications, and a taller than wide shape $[19,20]$.

2.2. US-Guided FNAB Procedure. US-guided FNABs were performed by three interventional radiologists with 6,8 , and 10 years of experience in thyroid biopsy, respectively. All biopsies were performed with US guidance by using a $5-12 \mathrm{MHz}$ linear probe (EPIQ7 or iU 22; Phillips Medical Systems, Bothell, WA, USA). The skin was aseptically prepared and draped, and approximately $1 \mathrm{~mL}$ of $1 \%$ lidocaine was injected for local anesthesia. US-guided FNABs were performed using a 22- or 23-gauge needle (Hakko, Nagano, Japan) with a free-hand biopsy technique. The biopsy approach for FNAB was used with either a parallel or perpendicular needle path to the probe (Figure 1 and 2). When the needle tip had reached the nodule, tissue samples were

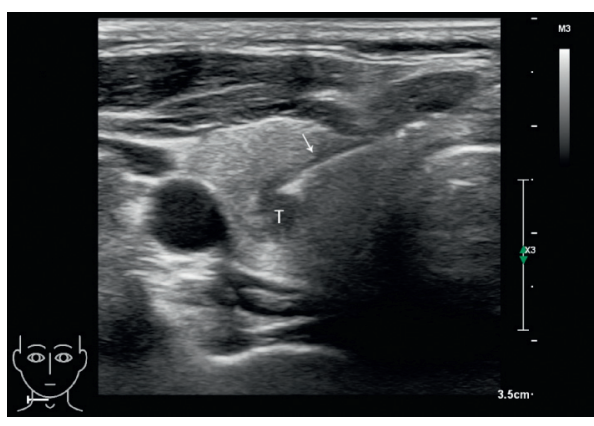

FIgURE 1: Parallel positioning of the fine needle (arrow) for thyroid nodule $(\mathrm{T})$ biopsy.

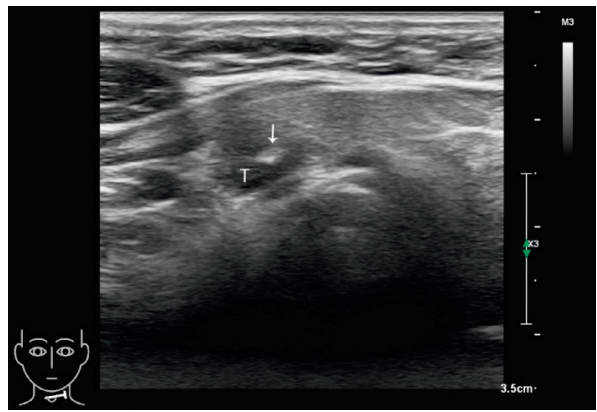

FIgURe 2: Perpendicular positioning of the fine needle (arrow) for thyroid nodule $(\mathrm{T})$ biopsy.

collected by using 6 to 7 to-and-fro needle motions over 5-10 seconds within the nodule, and according to the features of thyroid nodules, a combination of the aspiration and the capillary techniques was used. A total of 4 to 6 slides were prepared from the 3 to 4 passes for each of the nodules being biopsied. The specimens were immediately smeared on the glass slides band and then fixed with 95\% alcohol.

2.3. Cytologic Diagnosis. The specimens were classified as follows according to the Bethesda categories: (I) nondiagnostic; (II) benign; (III) atypia or follicular lesion of undetermined significance (AUS/FLUS); (IV) follicular neoplasm or suspicious for follicular neoplasm (FN/SFN); (V) suspicious for malignancy (SM); and (VI) malignant [21]. Categories II-VI were interpreted as diagnostic results.

The final diagnoses of malignant nodules (including SM) were confirmed by surgery. The final diagnoses of benign nodules were also confirmed by surgery, with clinical and imaging follow-up of at least 1 year.

2.4. Data Collection. Nodule-related factors included nodule size, nodule location, and ultrasound features. Nodule size was measured along the maximal diameter. Ultrasound features for the nodules were described for the following features: internal composition (solid, mixed, or cystic), shape (ovoid to round, taller than wide, or irregular), margin (defined or ill-defined), echogenicity (hypoechoic, isoechoic, or hyperechoic), and the presence of microcalcifcation or 
macrocalcifcation $[19,20]$. Technical factors included needle path, individual radiologist, and biopsy needle gauge.

2.5. Statistical Analysis. The chi-square test or Fisher exact test was used for the categorical variables. The two-tailed $t$-test was used to analyze the mean of a continuous variable. Multivariate logistic regression analysis was performed to select statistically significant variables with $P<0.05$ in univariate analysis. Statistical analyses were performed by using SPSS software ( version 17, SPSS Inc, Chicago, IL ), and a $P<0.05$ was considered as a statistically significant difference.

\section{Results}

3.1. Cytological Results and Surgical Results. There were 66 (18.9\%) nondiagnostic nodules, $46(13.2 \%)$ benign nodules, 34 (9.7\%) AUS/FLUS nodules, 12 (3.4\%) FN/SFN nodules, 91 (26.1\%) SM nodules, and 100 (28.7\%) malignant nodules (Figure 3). The adequate sample rate was $81.1 \%(283 / 349)$.

Surgical resection was performed in $66.8 \%$ (233 of 349) of the cases. There were 206 malignant nodules and 27 benign nodules. Out of the 283 cases with diagnostic results, $73.5 \%$ (208 of 283) of cases underwent surgery. Among them, 192 were malignant and 16 were benign (Table 1). Among the 25 cases with nondiagnostic results, there were four nodular goiters, seven follicular adenomas, and 14 papillary carcinomas (Table 1 ).

\subsection{Adequate Sample Rate in Relation to Nodule-Related} Factors. In regard to nodule size, the mean size of the 349 nodules was $7.5 \pm 1.9 \mathrm{~mm}$ (range $3-10 \mathrm{~mm}$ ); $31.5 \%$ (110 of 349) of nodules measured between 3 and $6 \mathrm{~mm}$ and $68.5 \%$ (239 of 349) of nodules measured between 7 and $10 \mathrm{~mm}$. In univariate analysis, the adequate sample rate increased with larger nodules; it was $72.7 \%$ (80 of 110$)$ for 3-6-mm nodules and $84.9 \%$ (203 of 239 ) for $7-10 \mathrm{~mm}$ nodules $(P=0.007)$ (Table 2). The adequate sample rate in nodules without calcification was significantly higher than that of nodules with either microcalcification ( $90.6 \%$ vs. $81.3 \% ; P=0.035)$ or macrocalcification $(90.6 \%$ vs. $63.9 \%$; $P>0.001)$. Furthermore, there was also significant difference between nodules with microcalcification and macrocalcification $(P=0.005)$ (Table 2$)$. In regard to other nodule-related factors, there was no significant difference in the adequate sample rate on the basis of nodule location, composition, shape, margin, and echogenicity.

3.3. Adequate Sample Rate in Relation to Technical Factors. For the FNAB, a 23-gauge needle was routinely used $(75.1 \%$ (262 of 349)) and a 22-gauge needle was selectively used (24.9\% (87 of 349$)$ ). The adequate sample rate was $82.1 \%$ (215 of 262) for biopsies performed with a 23-gauge needle and $78.2 \%$ (68 of 87 ) for biopsies performed with a 22 -gauge needle. There was no significant difference between the 23and 22-gauge needle $(P=0.421)$. The adequate sample rate of the specimens sampled by using the parallel needle path was significantly higher than that using the perpendicular needle path $(85.0 \%$ vs. $71.8 \% ; P=0.004)$ (Table 3$)$. No significance was found in the adequate sample rate in relation to an individual radiologist $(P=0.331)$.

3.4. Multivariate Statistical Analysis. Because some factors may explain other factors, leading to indirect bias, odds ratios (OR) were estimated for diagnostic yield by using multivariate logistic regression. The significant candidate variables were (a) nodule size, (b) calcification, and (c) needle path.

Regarding nodule size, there was an approximately 3.0 times higher likelihood of having a diagnostic result $(P<0.001 ; \quad O R=2.96 ; 95 \%$ confidence interval $(\mathrm{CI})$ : 1.63-5.37) with each increase in nodule size subgroup (3-6 mm, 7-10 mm) (Table 4). For calcification, the highest adequate sample rate correlated with a nodule without calcification, which was approximately 5.3 times more likely than a nodule with macrocalcification to achieve a diagnosis $(P<0.001 ; \mathrm{OR}=5.31 ; 95 \% \mathrm{CI}: 2.22-12.68)$ (Table 4). For needle path, the use of a parallel needle path was about 1.8 times more likely than with the use of a perpendicular needle path to achieve a diagnosis $(P=0.044$; $\mathrm{OR}=1.84 ; 95 \% \mathrm{CI}$ : 1.02-3.33) (Table 4).

\section{Discussion}

Thyroid nodules are common, and thyroid cancer is currently the fifth most common cancer in women [22]. With the improvement of high-frequency ultrasound, the detection rate of subcentimeter thyroid nodules has been significantly improved. The management of thyroid nodules should consider various factors, and cytological diagnosis of FNAB is the most important determinant in decision making [23]. At our institution, all patients underwent US assessment of the thyroid nodules according to the Thyroid Imaging Reporting and Data System (TI-RADS). Patients with subcentimeter thyroid nodules with suspicious US features (TI-RADS category $4 \mathrm{~A}$ and higher) were recommended for US-guided FNAB. The previous studies have reported that FNAB is still an effective tool for subcentimeter nodules $[7,10,13,24]$. However, one of the limitations of FNAB is nondiagnostic results due to insufficient sampling. Our results revealed that the adequate sample rate was $81.1 \%$, and 66 nodules $(18.9 \%$ ) yielded insufficient specimens on initial US-guided FNAB, which was similar to that reported in previous studies [7, 13, 16].

Nodule size is a determining factor for the adequate sample rate. The present study revealed that there was an increase in adequate sample rate as thyroid nodules become larger, which may be related to the difficulty in targeting smaller nodules. The adequate sample rate for the $7-10 \mathrm{~mm}$ nodules was significantly higher than that for the $3-6 \mathrm{~mm}$ nodules. Moon et al. [16] found that with the increase of nodule size, the inadequate sample rate decreased, and the inadequate sample rate was $20.8 \%$ for the $2-6 \mathrm{~mm}$ nodules and $15.3 \%$ for the nodules larger than $6 \mathrm{~mm}$ to $10 \mathrm{~mm}$ in diameter $(P=0.007)$. Kim et al. $[13]$ reported a significant difference in the adequate sample rate between the nodules 


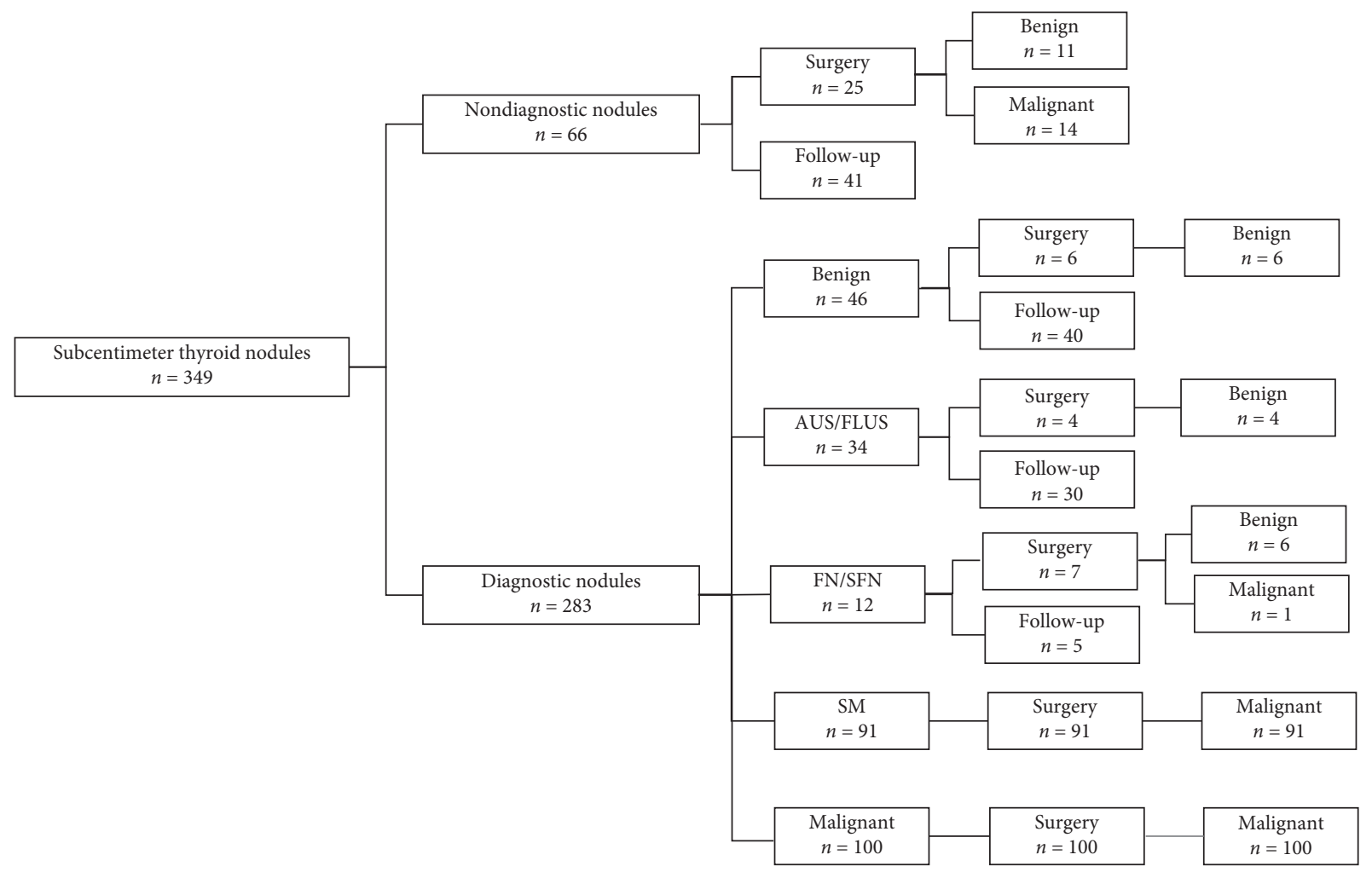

Figure 3: Flowchart showing the study population of 344 patients with 349 subcentimeter thyroid nodules.

TABLE 1: Histologic diagnosis after subsequent surgery in 233 nodules.

\begin{tabular}{lcc}
\hline Diagnosis at FNAB & No. of cases & Surgical diagnosis \\
\hline Nondiagnostic & 25 & Four nodular goiters, seven follicular adenomas, 14 papillary carcinomas \\
Benign & 6 & One adenomatous hyperplasia, two thyroiditis, three nodular goiters \\
AUS/FLUS & 4 & Two follicular adenomas, two adenomatous hyperplasias \\
FN/SFN & 7 & One papillary carcinoma, six follicular adenomas \\
SM & 91 & 91 papillary carcinomas \\
Malignant & 100 & Two medullary carcinoma, 98 papillary carcinomas \\
\hline
\end{tabular}

equal to or less than $5 \mathrm{~mm}$ and the nodules larger than $5 \mathrm{~mm}$ to $10 \mathrm{~mm}$ in diameter $(P=0.014)$. Contrary to the findings reported above, the study by Zhong et al. [7] showed that the nodule size did not appear to influence adequate sample rate and there was no significant difference between thyroid nodules $\leq 5 \mathrm{~mm}(n=56$; adequate sample rate, $82.2 \%)$ and nodules $6-10 \mathrm{~mm}(n=136$; adequate sample rate, $87.5 \%)$; furthermore, there were no statistical differences in the diagnostic sensitivity, specificity, positive predictive value, and negative predictive value of FNAB for thyroid nodules with different sizes. However, Kim et al. [13] found the sensitivity for nodules equal to or less than $5 \mathrm{~mm}$ was significantly lower than that for the nodules larger than $5 \mathrm{~mm}$ to $10 \mathrm{~mm}$. Dong et al. [8] reported that the nodules equal to or less than $5 \mathrm{~mm}$ with unclear borders were prone to yielding false positive results.

As for calcification, our study found that the inadequate sample rate of the nodules with macrocalcification was significantly higher than that of nodules with microcalcification or no calcification, which is in accordance with the result obtained by Choi et al. [25]. However, Grani et al. [26] reported that neither micro- nor macrocalcification increased the risk of inadequate sampling. Our results suggested that the highest adequate sample rate correlated with the nodules without calcification, which was approximately 5.3 times more likely to achieve a diagnosis as compared to the nodules with macrocalcification. We hypothesize that the high inadequate sample rate of nodules with macrocalcification may be related to the acquisition of limited solid areas of tumor cells during FNAB. First, it may be difficult to penetrate dense calcification with a fine needle. Second, due to strong posterior acoustic shadowing, the internal content and posterior edge of the nodule cannot be viewed, and US has a limitation in the evaluation of a nodule with macrocalcification. Therefore, the ultrasound features of macrocalcification cannot exclude the presence of a solid component hiding behind the posterior acoustic shadowing. We suggest radiologists should carefully analyze the US or 
TABLE 2: Adequate sample rate in relation to nodule-related factors.

\begin{tabular}{|c|c|c|c|c|}
\hline Variable & No. of adequate sampling cases & Total no. of cases & Adequate sample rate (\%) & $P$ value \\
\hline Nodule size $(\mathrm{mm})$ & & & & 0.007 \\
\hline $3-6$ & 80 & 110 & 72.7 & \\
\hline $7-10$ & 203 & 239 & 84.9 & \\
\hline Nodule location & & & & 0.916 \\
\hline Right & 141 & 172 & 82.0 & \\
\hline Isthmus & 8 & 10 & 80 & \\
\hline Left & 134 & 167 & 80.2 & \\
\hline Composition & & & & 0.312 \\
\hline Solid & 269 & 329 & 81.8 & \\
\hline Mixed & 14 & 20 & 70 & \\
\hline Shape & & & & 0.244 \\
\hline Ovoid to round & 84 & 110 & 76.4 & \\
\hline Taller than wide & 56 & 65 & 86.2 & \\
\hline Irregular & 143 & 174 & 82.2 & \\
\hline Margin & & & & 0.589 \\
\hline Defined & 100 & 121 & 82.6 & \\
\hline Ill-defined & 183 & 228 & 80.3 & \\
\hline Echogenicity & & & & 0.705 \\
\hline Hypoechoic & 248 & 304 & 81.6 & \\
\hline Isoechoic & 25 & 33 & 75.8 & \\
\hline Hyperechoic & 10 & 12 & 83.3 & \\
\hline Calcification & & & & $<0.001$ \\
\hline None & 96 & 106 & 90.6 & \\
\hline Microcalcification & 148 & 182 & 81.3 & \\
\hline Macrocalcification & 39 & 61 & 63.9 & \\
\hline
\end{tabular}

${ }^{a}$ A statistically significant difference in diagnostic yield between nodules without calcification and nodules with either microcalcification $(P=0.035)$ or macrocalcification $(P<0.001)$ and nodules with microcalcification and macrocalcification $(P=0.005)$.

TABle 3: Adequate sample rate in relation to technical factors.

\begin{tabular}{|c|c|c|c|c|}
\hline Variable & No. of adequate sampling cases & Total no. of cases & Adequate sample rate (\%) & $P$ value \\
\hline Biopsy needle gauge & & & & 0.421 \\
\hline 22 & 68 & 87 & 78.2 & \\
\hline 23 & 215 & 262 & 82.1 & \\
\hline Needle path & & & & 0.004 \\
\hline Perpendicular & 74 & 103 & 71.8 & \\
\hline Parallel & 209 & 246 & 85.0 & \\
\hline Radiologist & & & & 0.331 \\
\hline A & 94 & 115 & 81.7 & \\
\hline $\mathrm{B}$ & 99 & 128 & 77.3 & \\
\hline $\mathrm{C}$ & 90 & 106 & 84.9 & \\
\hline
\end{tabular}

TABLE 4: Adequate sample rate according to various related factors evaluated by multivariate logistic regression.

\begin{tabular}{|c|c|c|c|c|}
\hline Variable & Adequate sample rate (\%) & $P$ value & OR & $95 \% \mathrm{CI}$ \\
\hline \multicolumn{5}{|l|}{ Nodule size $(\mathrm{mm})$} \\
\hline $3-6$ & $72.7(80 / 110)$ & Reference & 1.0 & \\
\hline $7-10$ & $84.9(203 / 239)$ & $<0.001$ & 2.96 & $1.63-5.37$ \\
\hline \multicolumn{5}{|l|}{ Calcification } \\
\hline None & $90.6(96 / 106)$ & $<0.001$ & 5.31 & $2.22-12.68$ \\
\hline Microcalcification & $81.3(148 / 182)$ & 0.001 & 3.20 & $1.61-6.37$ \\
\hline Macrocalcification & $63.9(39 / 61)$ & Reference & 1.0 & \\
\hline \multicolumn{5}{|l|}{ Needle path } \\
\hline Perpendicular & $71.8(74 / 103)$ & Reference & 1.0 & \\
\hline Parallel & $85.0(209 / 246)$ & 0.044 & 1.84 & $1.02-3.33$ \\
\hline
\end{tabular}


CT imaging of nodules with calcification and choose best location from which to get the sample.

Our results indicated that the use of parallel needle path for biopsying small $(\leq 10 \mathrm{~mm})$ thyroid nodules resulted in a higher adequate sample rate. To our knowledge, only one study has compared the effects of parallel and perpendicular techniques on specimen adequacy from thyroid nodules and reported that the use of parallel needle path significantly decreased the inadequate specimens compared to use of perpendicular needle path; however, there were only 80 nodules in this study [27]. Our clinical observation revealed that if the needle is parallel to the probe, the location of the tip and the entire pathway of the needle can be seen from the skin puncture to the nodule. The result that the parallel needle path approach enhances the specimen adequacy of FNAB depends also on better real time monitoring of the needle tip during the aspiration. However, if the needle is perpendicular to the probe, the needle tip is viewed only as a hyperechoic focus when it enters the nodule, and localization of the needle tip becomes more difficult.

Factors such as nodule location, composition, shape, margin, and echogenicity do not appear to affect the adequate sample rate. As for composition, we expected solid thyroid nodule to be a factor associated with high adequate sample rate. Choi et al. [25] reported that cyst-dominant nodules had a significantly higher inadequate sample rate than solid or solid dominant nodules. However, Grani et al. [26] reported that mixed nodules were more frequently diagnostic. In our study, there was no significant difference in the adequate sample rate between solid and mixed nodules. One possible reason for the result may be that the number of mixed lesions was only 20. Of the 20 mixed nodules, 19 were predominantly solid nodules and one was predominantly cystic nodule. We believe that subcentimeter thyroid nodules are less likely to develop cystic degeneration.

There were several potential limitations to our study. First, our study was of retrospective design. Second, there may be a selection bias, nodules were solid, micro- and macrocalcification very frequent, and the sample size of the mixed nodules and benign nodules is relatively small. Finally, the radiologists may choose different paths to puncture for a particular nodule, and furthermore, according to the professional level, the preference of the individual radiologist for a needle path is difficult to assess.

In conclusion, nodule-related and technical factors affecting adequate sample rate with US-guided FNAB of subcentimeter thyroid nodules were identified. The adequate sample rate was related to nodule size, calcification, and needle path. The results of our study revealed that the adequate sample rate was higher in larger nodules, in nodules without calcification, and upon use of the parallel needle path for biopsy.

\section{Data Availability}

The data that support the findings of this study are available from the corresponding author upon reasonable request.

\section{Ethical Approval}

This retrospective study was approved by the institutional review board.

\section{Conflicts of Interest}

The authors declare no conflicts of interest.

\section{Acknowledgments}

This study was supported by the Department of Education Fund (Grant nos. 18TD0028 and 17ZA0186) of Sichuan Province, the Bureau of Science and Technology and Intellectual Property, Nanchong city (Grant nos. NSMC20170431 and 18SXHZ0405), and the North Sichuan Medical College (Grant nos. CBY15-QD11).

\section{References}

[1] S.-Y. Kim, H. S. Lee, J. Moon et al., "Fine-needle aspiration versus core needle biopsy for diagnosis of thyroid malignancy and neoplasm: a matched cohort study," European Radiology, vol. 27, no. 2, pp. 801-811, 2017.

[2] M. J. Kim, E.-K. Kim, S. I. Park et al., "US-guided fine-needle aspiration of thyroid nodules: indications, techniques, results," Radiographics, vol. 28, no. 7, pp. 1869-1886, 2008.

[3] I. H. Chae, E.-K. Kim, H. J. Moon, J. H. Yoon, V. Y. Park, and J. Y. Kwak, "Ultrasound-guided fine needle aspiration versus core needle biopsy: comparison of post-biopsy hematoma rates and risk factors," Endocrine, vol. 57, no. 1, pp. 108-114, 2017.

[4] J. M. Chua, J. Y. Tang, D. S. Lim et al., "Should we perform fine needle aspiration cytology of subcentimetre thyroid nodules? A retrospective review of local practice," Ultrasound, vol. 27, no. 1, pp. 64-68, 2019.

[5] J. Luo, C. Zhang, F. Huang et al., "Risk of malignancy in thyroid nodules: predictive value of puncture feeling of grittiness in the process of fine-needle aspiration," Scientific Reports, vol. 7, no. 1, p. 13109, 2017.

[6] M. Shrestha, B. A. Crothers, and H. B. Burch, "The impact of thyroid nodule size on the risk of malignancy and accuracy of fine-needle aspiration: a 10-year study from a single institution," Thyroid, vol. 22, no. 12, pp. 1251-1256, 2012.

[7] L. C. Zhong, F. Lu, F. Ma et al., "Ultrasound-guided fineneedle aspiration of thyroid nodules: does the size limit its efficiency?" International Journal of Clinical and Experimental Pathology, vol. 8, no. 3, pp. 3155-3159, 2015.

[8] Y. Dong, M. Mao, W. Zhan et al., "Size and ultrasound features affecting results of ultrasound-guided fine-needle aspiration of thyroid nodules," Journal of Ultrasound in Medicine, vol. 37, no. 6, pp. 1367-1377, 2018.

[9] K. Kaliszewski, D. Diakowska, B. Wojtczak et al., "fine-needle aspiration biopsy as a preoperative procedure in patients with malignancy in solitary and multiple thyroid nodules," PLoS One, vol. 11, no. 1, Article ID e0146883, 2016.

[10] D. H. Koo, K. Song, H. Kwon et al., "Does tumor size influence the diagnostic accuracy of ultrasound-guided fine-needle aspiration cytology for thyroid nodules?" International Journal of Endocrinology, vol. 2016, no. 1, 6 pages, 2016.

[11] J. Y. Sung, D. G. Na, K. S. Kim et al., "Diagnostic accuracy of fine-needle aspiration versus core-needle biopsy for the 
diagnosis of thyroid malignancy in a clinical cohort," European Radiology, vol. 22, no. 7, pp. 1564-1572, 2012.

[12] S. H. Choi, J. H. Baek, J. H. Lee et al., "Thyroid nodules with initially non-diagnostic, fine-needle aspiration results: comparison of core-needle biopsy and repeated fine-needle aspiration," European Radiology, vol. 24, no. 11, pp. 2819-2826, 2014.

[13] D. W. Kim, E. J. Lee, S. H. Kim et al., "Ultrasound-guided fineneedle aspiration biopsy of thyroid nodules: comparison in efficacy according to nodule size," Thyroid, vol. 19, no. 1, pp. 27-31, 2009.

[14] B. Degirmenci, A. Haktanir, R. Albayrak et al., "Sonographically guided fine-needle biopsy of thyroid nodules: the effects of nodule characteristics, sampling technique, and needle size on the adequacy of cytological material," Clinical Radiology, vol. 62, no. 8, pp. 798-803, 2007.

[15] X. Liu, L. Zhu, Z. Wang et al., "Comparison of two different standards of care in detecting malignant thyroid nodules using thyroid fine-needle aspiration," Molecular and Clinical Oncology, vol. 3, no. 3, pp. 682-686, 2015.

[16] H. J. Moon, E. Son, E.-K. Kim, J. H. Yoon, and J. Y. Kwak, "The diagnostic values of ultrasound and ultrasound-guided fine needle aspiration in subcentimeter-sized thyroid nodules," Annals of Surgical Oncology, vol. 19, no. 1, pp. 52-59, 2012.

[17] E. L. Mazzaferri and J. Sipos, "Should all patients with subcentimeter thyroid nodules undergo fine-needle aspiration biopsy and preoperative neck ultrasonography to define the extent of tumor invasion?" Thyroid, vol. 18, no. 6, pp. 597-602, 2008.

[18] H. Gharib, E. Papini, R. Paschke et al., “American Association of Clinical Endocrinologists, Associazione Medici Endocrinologi, and European Thyroid Association medical guidelines for clinical practice for the diagnosis and management of thyroid nodules," Journal of Endocrinological Investigation, vol. 33, no. 5, pp. 1-50, 2010.

[19] A. Sharma, H. Gabriel, A. A. Nemcek, R. Nayar, H. Du, and P. Nikolaidis, "Subcentimeter thyroid nodules: utility of sonographic characterization and ultrasound-guided needle biopsy," American Journal of Roentgenology, vol. 197, no. 6, pp. W1123-W1128, 2011.

[20] W.-J. Moon, S. L. Jung, J. H. Lee et al., "Benign and malignant thyroid nodules: US differentiation-multicenter retrospective study," Radiology, vol. 247, no. 3, pp. 762-770, 2008.

[21] Z. W. Baloch, V. A. LiVolsi, S. L. Asa et al., "Diagnostic terminology and morphologic criteria for cytologic diagnosis of thyroid lesions: a synopsis of the national cancer institute thyroid fine-needle aspiration state of the science conference," Diagnostic Cytopathology, vol. 36, no. 6, pp. 425-437, 2008.

[22] B. R. Haugen, "American thyroid association management guidelines for adult patients with thyroid nodules and differentiated thyroid cancer: what is new and what has changed?" Cancer, vol. 123, no. 3, pp. 372-381, 2017.

[23] S. Tamhane and H. Gharib, "Thyroid nodule update on diagnosis and management," Clinical Diabetes and Endocrinology, vol. 2, no. 1, p. 17, 2016.

[24] S. J. Kim, E. K. Kim, C. S. Park, W. Y. Chung, K. K. Oh, and H. S. Yoo, "Ultrasound-guided fine-needle aspiration biopsy in nonpalpable thyroid nodules: is it useful in infracentimetric nodules?" Yonsei Medical Journal, vol. 44, no. 4, pp. 635-640, 2003.

[25] S. H. Choi, K. H. Han, J. H. Yoon et al., "Factors affecting inadequate sampling of ultrasound-guided fine-needle aspiration biopsy of thyroid nodules," Clinical Endocrinology, vol. 74, no. 6, pp. 776-782, 2011.

[26] G. Grani, A. Calvanese, G. Carbotta et al., "Intrinsic factors affecting adequacy of thyroid nodule fine-needle aspiration cytology," Clinical Endocrinology, vol. 78, no. 1, pp. 141-144, 2013.

[27] E. Kandil, M. A. Khalek, H. Alabbas et al., "Comparison of ultrasound-guided biopsy technique for thyroid nodules with respect to adequacy of cytological material," ORL, vol. 73, no. 4, pp. 177-181, 2011. 